\title{
The Potential of Antibiotic Alternatives for Enriching Beneficial Microbiota in Litter
}

\author{
Adriana A. Pedroso ${ }^{1}$, Stephen R. Collett ${ }^{1}$, Anne L. Hurley-Bacon ${ }^{2}$, Andrea S. Zedek ${ }^{3}$ and Margie \\ D. Lee ${ }^{1 *}$ \\ ${ }^{1}$ Poultry Diagnostic and Research Center, College of Veterinary Medicine, The University of Georgia, Athens, Georgia, United States of America \\ ${ }^{2}$ Merial Limited, Athens, Georgia \\ ${ }^{3}$ Zoetis Animal Health, Madison, New Jersey
}

Received: March 25, 2014; Accepted: June 08, 2014; Published: June 11, 2014

*Corresponding author: Margie D. Lee, Poultry Diagnostic and Research Center, 953 College Station Rd, Athens, GA 30677, USA, E-mail: mdlee@uga.edu

\begin{abstract}
Alternative growth promoters such as probiotics, prebiotics and competitive exclusion products have been used with some success in animal production. A compelling application of these products is their potential to reduce pathogen populations by altering the environmental bacterial community of a built up litter system over multiple grow-outs. The objectives of this study were determine the bacterial composition of the litter over multiple production cycles and describe the impact of alternative growth promoters on composition and abundance of bacteria viewed as beneficial or pathogenic. In this study, probiotics were found to have the ability to positively alter the litter bacterial community. Bacterial diversity and the proportion of the community consisting of beneficial bacteria were increased in the litter when probiotics were administered. As the litter aged, diversity decreased and the composition shifted from Firmicute to Actinobacteria. Detection of pathogenic Clostridium species increased in litter with time regardless of the type of product used. However, detection of Enterococcus cecorum decreased over time and treatments produced less E. cecorum in reused litter after 1 flock cycle. These findings suggest that administering alternatives to antibiotics can change the litter bacterial composition, increase the abundance of beneficial bacteria and reduce the prevalence of some pathogens.
\end{abstract}

Keywords: Microbiota; Microbiome; Litter; 16S; Broiler; Probiotic; Enterococcus; Microflora

\section{Introduction}

The gastrointestinal tract is home to a diverse population of microorganisms that can have a great impact on chick health, either positively or negatively. In a natural setting, gastrointestinal organisms are rapidly obtained from the egg shell and by consuming fecal material from the adult hen [1]. In today's commercial poultry industry however, the development of a complex intestinal microbiota is delayed because sanitation in commercial hatcheries have severed the connection between the chick and the complex fecal microbiota associated with the hen. This allows environmental bacteria to play a larger role in seeding the intestinal tract of the commercial broiler chick $[1,2]$. Many commercial poultry companies use a built up litter system, meaning that the bacteria left in the litter from the previous flock(s) could potentially have a major impact, positive or negative, on the gastrointestinal health status of the young broiler chick. Historically, commercial poultry producers have fed low levels of antibiotics in their poultry ration to negate the impact of pathogen growth on feed conversion ratios and weight gain; however, because of consumer pressure and government mandated restrictions on antibiotic usage, many poultry companies are establishing antibiotic-free programs $[3,4]$ demonstrating increasing interest in the development of alternatives to low dosage antibiotic usage in the poultry industry.

Probiotics, competitive exclusion products and prebiotics are all potential antibiotic alternatives to help alleviate the cost of enteric disease in poultry production. Some antibiotic alternatives have been shown to reduce pathogens in the intestinal tract of poultry resulting in increased weight gain, feed conversion ratio, and livability [4-8]. Probiotics are live microbial feed supplements designed to benefit the host by improving the intestinal microbial ecology [9]. They have been utilized for centuries, but a scientific hypothesis regarding their benefits was not proposed until the turn of the $20^{\text {th }}$ century when Elie Metchnikoff observed the unusually long life span of Bulgarians that consumed fermented milk [10]. In 1907 he proposed that the ingestion of lactic acid producing bacteria found in fermented milk had a positive impact on health $[11,12]$. The exact mechanism through which these products provide benefits is being studied and may actually differ depending on the specific formulation. Mechanisms include modifying the $\mathrm{pH}$ of the gastrointestinal tract, production of antimicrobial or signaling molecules, competing for ecological niches and available nutrients, promoting epithelial cell differentiation and turnover, affecting mucus production, aiding in the metabolism of nutrients by the host, and/or stimulation of the immune system [13-17]. An exciting new perspective on intestinal/microbial symbiosis comes from the finding that pioneer colonizers, the first bacteria to reach the neonatal gut, can directly influence the development of the intestine and the nutrient matrix it provides for sequential bacterial species that eventually comprise the complex microbiota $[18,19]$. This is not only important for optimal gut development [19] but for disease 
resistance [20-25] and modulation of inflammation [26-32]. It is imperative that chicks receive autogenous pioneer colonizers to mediate the development of a healthy intestinal microbiome essential for optimal flock performance. There are many commercially available probiotic and competitive exclusion products that contain autogenous pioneer colonizers.

Prebiotics are defined as "a non-digestible food ingredient that beneficially affects the host by selectively stimulating the growth and/or activity of one or a limited number of bacteria in the intestine [15]. Prebiotics contain complex carbohydrates that pass through the small intestine to the lower gastrointestinal tract where they are utilized by specific bacteria [15]. Feed containing these indigestible complex carbohydrates affect the composition of intestinal microbiota because the status of the intestinal food web is altered. Variations in the available carbon and energy sources affects the abundance of primary degraders of particular substrates and modify the proportion of scavenger microorganisms that are able to utilize the metabolic by-products produced by the primary degraders. These interactions generate changes in the composition and metabolism of the microbiome and can tip the balance towards a more beneficial profile. Galactoand fructo- and mannanoligosaccharides are the most commonly used prebiotics. Mannanoligosaccharides have demonstrated a number of benefits in poultry including increasing abundance of lactobacilli and bifidobacteria, binding of type-1fimbriae on gram-negative bacteria which reduces abundance, modulating the immune response, and reducing the enterocyte turnover rate $[15,33]$.

Since Metchnikoff's observations, numerous studies have shown that a fermentative microbiota can inhibit pathogens, that disturbances of the intestinal microbiota can increase susceptibility to infection and that addition of prebiotics and probiotics increase resistance to infection $[11,20]$. One of the most important studies by Nurmi and Ratali [17] demonstrated that day old chicks were protected against colonization by Salmonella following the administration of an oral suspension of cecal contents from an adult chicken [2]. The "Nurmi concept" is better known as competitive exclusion and exploited worldwide to reduce pathogen colonization of humans and animals [1,2,34]. However, because competitive exclusion products are made from the microbiota of adult animals, they also contain pioneer colonizers that enhance development of the intestine.

An effective intestinal health program for poultry production includes "seeding" the gut of hatchlings with favorable organisms (probiotics or competitive exclusion products), "feeding" the favorable organisms with prebiotics and organic acids and "weeding" out the unfavorable organisms with products such as type-1 fimbria blockers [35]. The "seed, weed, feed" concept for utilizing antibiotic alternatives in poultry production is most effective if it is applied to sequential flocks. Reports from the field indicate that several flock cycles may be needed in order to improve production parameters and resolve recurrent disease houses/farms. This observation indicates that the litter microbiota is important in developing a healthy intestinal microbiota. While there have been many cultivation-based studies focusing on the presence of specific bacteria in chicken litter [36-39], it has been difficult to thoroughly characterize the composition of litter due to inadequate traditional culture techniques. Selective media is often not as specific in some instances and too selective to allow certain bacteria to be detected. However the litter microbiota has been characterized by molecular ecology techniques in a few studies $[17,40]$ and recently we reported the effects of probiotics or prebiotics on the microbiome and antibiotic resistome of broiler litter [42]. The objective of this report was to evaluate the impact of alternative growth promoters on the litter microbiota from a chick quality standpoint.

\section{Material and Methods \\ Housing, litter and treatments}

Five colony houses equipped with fresh pine shaving litter were top-dressed with 20 gallons/house of built-up litter obtained from a Northeast Georgia commercial broiler house. Five hundred day-of-age broiler chicks were obtained from a commercial hatchery, and 100 chicks were randomly allocated to each of the colony houses at a stocking density of $1 \mathrm{ft}^{2} /$ bird. Birds were grown until the end of the rearing period and received All-Lac (Lactobacillus, Enterococcus, and Pediococcus Alltech Inc, Lexington KY USA) the combination of All-Lac and Biomos (Mannanoligosaccharide - Alltech Inc, Lexington KY USA), Aviguard (Undefined bacteria collected and cultured from chicken cecum content - Microbial Developments Ltd Malvern UK) and Primalac (Lactobacillus spp, Enterococcus faecium, and Bifidobacterium thermophilum - Star-Labs, Clarksdale MO USA) as per the manufacturer's directions; the control group did not receive any treatment [41]. After down time, each colony house was repopulated with day-old chicks from the same commercial hatchery, the designated probiotic product was applied for each treatment group again and this cycle was repeated for a total of 4 production cycles on the same litter throughout the study. Each flock was reared for 6 weeks; no litter was collected when birds were not in the flock house. Weekly when the birds were present, five random litter samples from each colony house were obtained using a number/grid system and pooled. A 5 gram sub-sample was then taken from the pool and mixed with $20 \mathrm{ml}$ of $0.9 \% \mathrm{NaCl}$, homogenized for two minutes and stored at $-80^{\circ} \mathrm{C}$ for further analysis.

\section{Animal performance}

At the end and beginning of each cycle the birds were weighed. Mortality of birds was recorded daily and used to calculate the percentage of dead birds for each cycle. A standard commercial broiler diet based on corn-soy meal containing only monensin as anticoccidia was supplied ad libitum and the amount supplied for each house recorded. Feed conversion ratio, live weight, and total mortality were determined at the end of the second, third and fourth cycle.

\section{Characterization of litter bacterial composition}

The protocol used for isolating the community DNA from litter samples was as previously described [40]. PCR amplification of the V3 and $\mathrm{V} 6$ region of the bacterial 16S rRNA genes was 
conducted as previously described [42]. DNA extracted from Salmonella typhimurium SR11 was used as control to test for errors during PCR amplification and pyrosequencing. Samples were submitted to the University of Georgia Genomics Facility for pyrosequencing according to methods established by the manufacturer. Sequences analysis was carried out using MOTHUR v1.21.0 [43]. Good quality sequences were aligned using the SILVA database in MOTHUR and further filtered. Distances were calculated on preclustered sequences, and operational taxonomic units (OTU) were formed using the average neighbor method in MOTHUR.

\section{Statistical analysis}

Statistical treatment related to the frequency of specific sequences present in samples, and animal performance were conducted by analysis of variance using the general linear procedure of SAS software [44]. Tukey test was used to test differences $(\mathrm{P} \leq 0.05)$.

\section{Results and Discussion}

Poultry deep litter systems have been used as a practice to improve efficiency of meat production and to decrease environmental waste, however little is known about the microbial composition of the reused litter over time. Correct management of poultry litter is important for good animal indexes because it affects air quality and pathogen load in the poultry house. However it can act as a source of beneficial organisms such as pioneer colonizers that stimulate development of the chicken intestinal tract and competitive exclusion bacteria to reduce the prevalence of food safety pathogens. In this study, we generated large scale 16S rRNA libraries of litter samples produced during four cycles of poultry production supplemented with alternative growth promoters to investigate changes in bacterial composition and frequency of pathogens [41].

\section{Flock performance}

Mean body weights ranged from 1744-1875 grams with no statistical differences among cycles or treatments. There were also no statistically significant differences in feed conversion ratios (2.05-2.22), or mortality rates (2.2-6.3\%) among the treatments or the cycle of production. These birds were reared with low density in research colony houses which allowed for low stress and disease prevalence.

\section{Phylogeny of the sequences obtained}

After processing of sequences for quality and length, the 64 libraries produced 367,688 total sequences [41]. Seven samples failed to generate a significant number of reads, thus were removed from the analysis. The genera that contributed to at least $1 \%$ of the sequences were primarily from the phyla Firmicutes and Actinobacteria. Firmicutes and Actinobacteria are grampositive organisms common in soil and compost. Despite the variation in number of sequences from each sample, Firmicutes contributed an average of $65 \%$ while Actinobacteria contributed $28 \%$. The most abundant bacteria within these phyla were Corynebacterium casei, C.ammoniagenes, Staphylococcus cohnii, S. saprophyticus, S. arlettae, Salinicoccus sp., Brachybacterium paraconglomeratum, Brevibacterium linens, Jeotgalibacillus marinus, and Yaniella sp. However a number of intestinal bacteria including multiple species of Lactobacillus, Enterococcus, and Clostridia were also detected.

\section{Effect of time and treatments on composition}

Overall composition of litter at the phylum level for each treatment is shown in (Figure 1). The proportions of Firmicutes and Actinobacteria were similar among treatments however samples from All-Lac and Primalac treated flocks contained a higher proportion of other bacteria. The control group exhibited the least diversity while Primalac or All-Lac + BioMos treatments produced the most diverse litter samples (Figure 2 and 3). Primalac or All-Lac + BioMos treatment also resulted in more genera detected in the litter (higher richness) and these samples exhibited higher Shannon diversity indices among the samples. Diversity decreased over time among all treatments which indicates that they have the ability to impact composition of the litter bacterial community.

Figure 4 shows the effects of the treatments on the litter composition over time. The first sample from all groups was dominated by the phylum Firmicutes. In the control group and All-Lac + BioMos group, Staphylococcus was the most abundant while all other groups were dominated by Salinococcus. The most abundant phylum in the last sample was Firmicutes in all treatment groups except the treatment group receiving All-Lac + BioMos, which was dominated by the phylum Actinobacteria. Overtime, the control group and treatment groups receiving All-Lac or Aviguard saw an increase in Actinobacteria including Corynebacterium, Brachybacterium, and Brevibacterium. Members of the Actinobacteria are likely involved in the decomposition of organic material like wood shavings [45]. They have been found to be key microbes for composting of litter in culture-based studies $[37,46]$ and the shift in bacterial communities over multiple production cycles is not unexpected given the physical differences between fresh and used litter.

Intestinal bacterial species are components of some of the products used in this study. All-Lac consists of Lactobacillus, Enterococcus, and Pediococcus; Primalac contains Lactobacillus species, Enterococcus, and Bifidobacterium. Aviguard is a fermented product from chicken cecal microbiota. (Figure 4, 5) show the effects of treatments on the abundance of potential intestinal symbiotic bacteria. In the control group Lactobacillus was seen to increase in abundance during an individual cycle, however over multiple production cycles abundance decreased as the flock aged. This trend was not seen in the treatment groups; Lactobacillus tended to be higher at the start of an individual cycle than at the end of the grow out cycle and there was no overall decreasing trend in the Lactobacillus abundance over multiple production cycles in any of the flocks treated with antibiotic alternatives. Litter from Primalac, All-Lac, and Aviguard treated flocks had higher abundance of Enterococcus while all treatments elicited higher abundance of intestinal bacteria than control. Among the bacteria commercially available as probiotics, Lactobacillus and Enterococcus were detected in every litter 


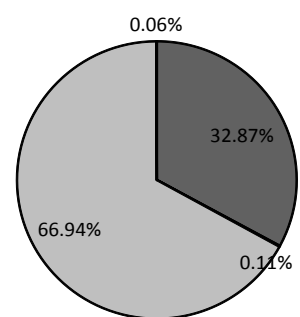

Control

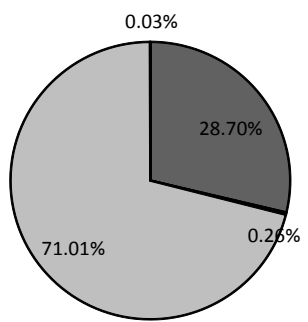

Aviguard

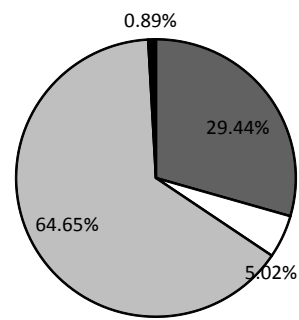

All-Lac

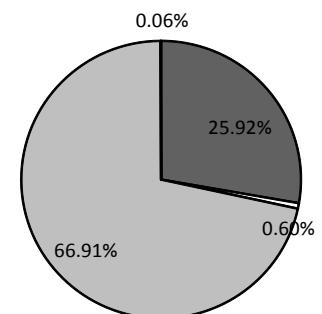

Primalac

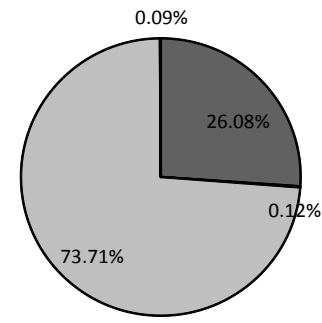

All-Lac + BioMos

Figure 1: Overall composition of the litter at the phylum level.

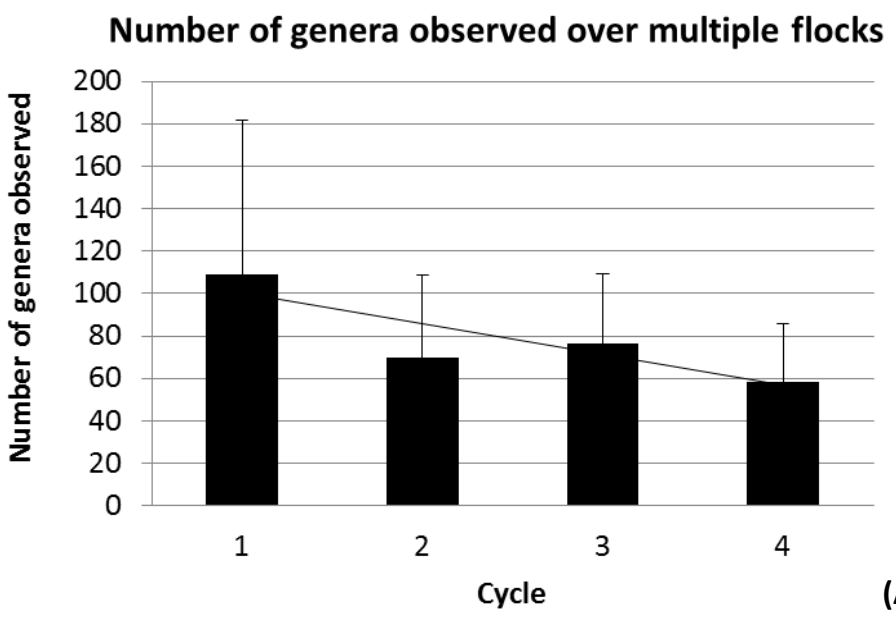

-Actinobacteria
$\square$ Proteobacteria
$\square$ Firmicute
- Other

\section{Number of genera observed among treatments}

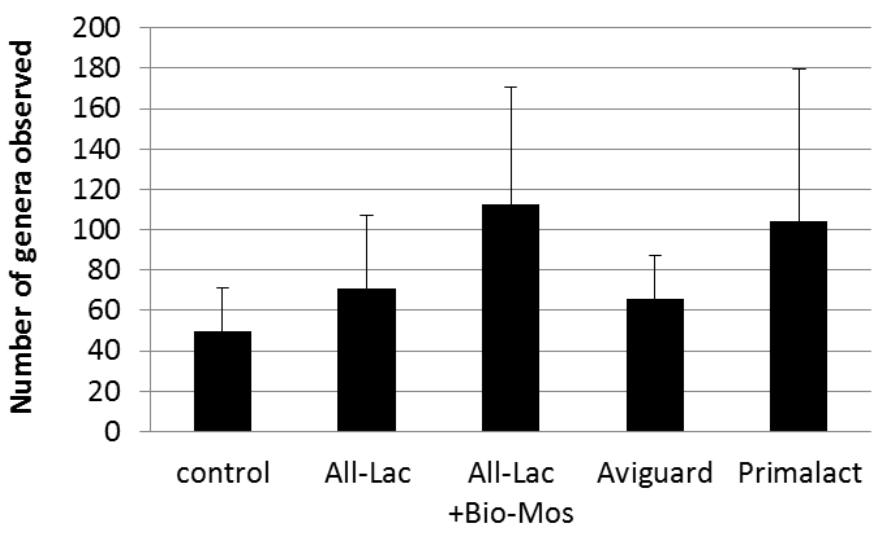

(B)

Figure 2: Diversity of the litter microbiota over time (A) and flock treatments (B). 


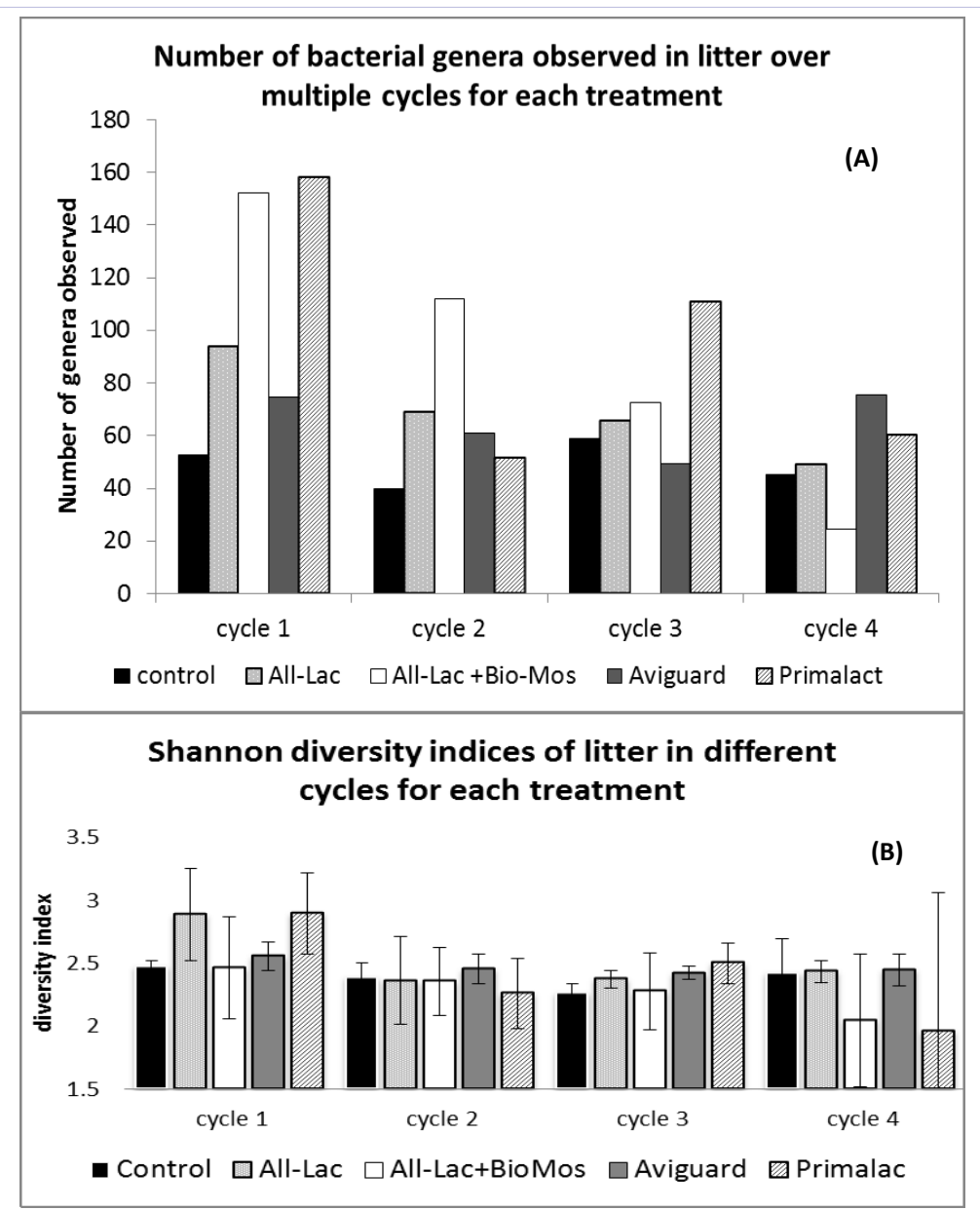

Figure 3: Effect of treatments on the composition (A) and diversity (B) of the litter microbiota over multiple flocks.

sample but other probiotic bacteria occurred in low abundance. Bacillus was sporadically detected and occurred primarily in the early litter samples. Bifidobacterium was detected in low abundance in many litter samples from the flocks treated with Aviguard but primarily in the later samples in the Primalac group.

\section{Effect of time and treatments on abundance of pioneer colonizers}

The bacterial community of the first sample from the first cycle was statistically different $(\mathrm{P}<0.05)$ than the community within the last sample from the fourth cycle for all groups. The fact that the control house was statistically different indicates that time does have a significant impact on the microbiota. The top portion of (Figure 4) shows the proportion of intestinal anaerobic bacteria, such as Atopostipes and Clostridium cluster $\mathrm{XI}$, increases over time. There is a current belief that old litter from chickens harbors a seed intestinal community of pioneer colonizers that can be transmitted to the next poultry flock. We hypothesize that litter from flocks treated with alternatives to antibiotics may have an enriched community of pioneer colonizers. In order to investigate this hypothesis, we evaluated the composition of the libraries in regards to the "rare biosphere" [47]. The library of litter sequences contained total of 1103 operational taxonomic units at the species level however the majority of these contributed less than 10 sequences and in many cases only one or two sequences were detected. Figure 4 and Table 1 shows that the abundance of the rare biome is significantly different over time and with treatments. While the overall percentage of total sequences represented by the rare biome was not large, the number and types of sequences that comprised the rare biome was very different. Potential intestinal symbionts, which would be transmitted from flock to flock, would likely be present within this less abundant biosphere. To test this hypothesis we looked for microorganisms that could be used as markers such as members of the intestinal symbiotic Clostridia that would be increasing in abundance in the control group but abundant in the treated groups. We found that sequences with similarity to Candidatus Arthromitus sp. SFB-mouse, Clostridium aminophilum, C. butyricum, C. cellobioparum, C. cylindrosporum, C. hylemonae, C. limosum, C. scindens, Clostridium sp. Sukashi-1, Clostridium sp. enrichment culture clone 7-14, C. tyrobutyricum, Finegoldia magna, Tissierella creatinophila, Eubacterium coprostanoligenes, E. fissicatena, E. hallii, Hespellia porcina, Roseburia faecis, Ethanoligenens harbinense, Faecalibacterium prausnitzii,Ruminococcus callidus, R. flavefaciens, R. gauvreauii, R. obeum, Blautia producta, B. hydrogenotrophica, and butyrate- 

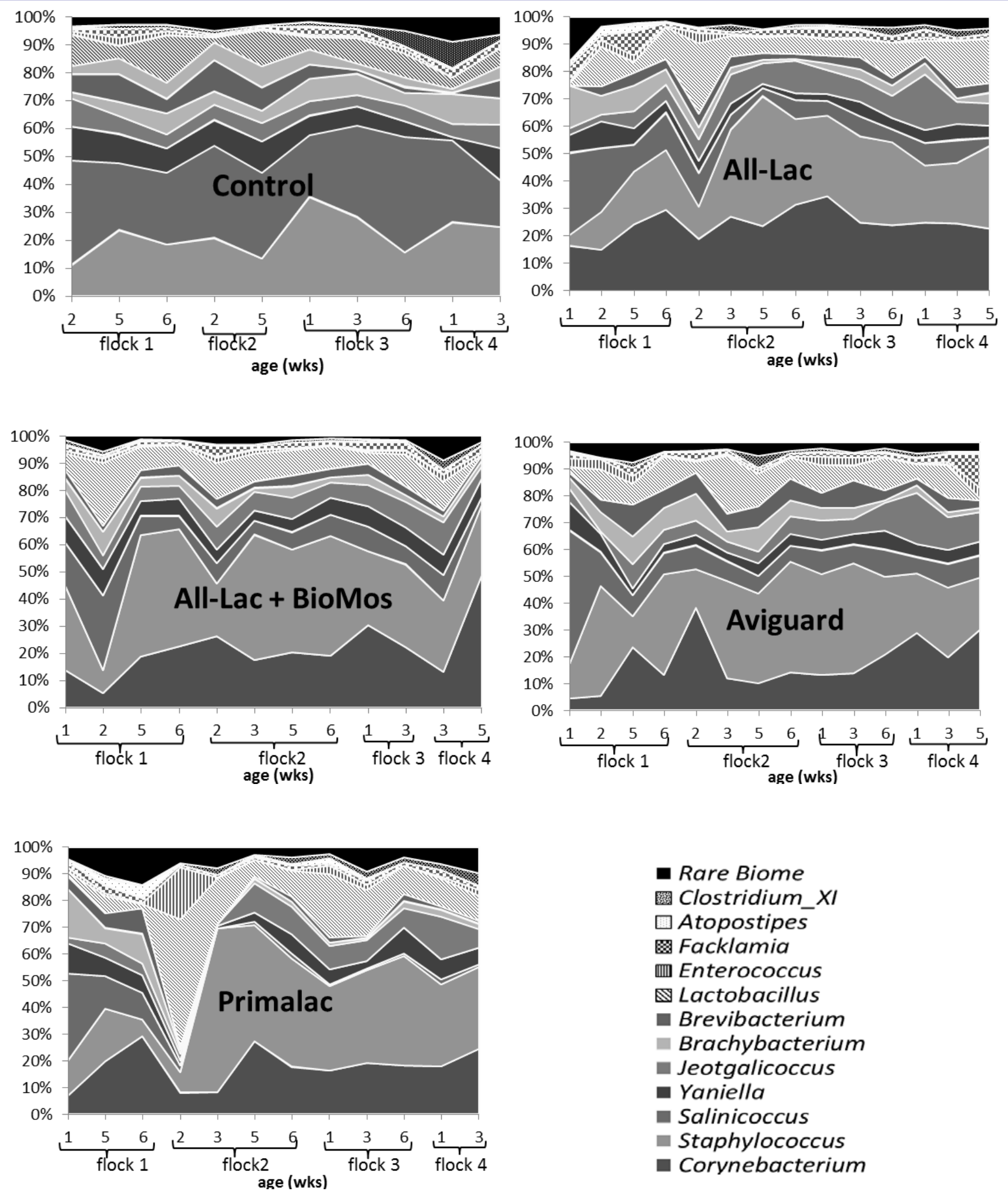

Figure 4: Effect of treatments on the composition of the litter microbiota over time. Distribution of bacterial abundance is shown on Y-axis. Intestinal bacterial, at the top of the graphs, are shown as patterned areas; environmental bacteria are shown at the bottom; organisms occur in the graphs in the order in which they are listed in the legend.

Citation: Pedroso AA, Collett SR, Hurley-Bacon AL, Zedek AS, Lee MD (2014) The Potential of Antibiotic Alternatives for Enriching Page 6 of 11 Beneficial Microbiota in Litter. SOJ Microbiol Infect Dis 2(2): 1-11. DOI: http://dx.doi.org/10.15226/sojmid.2014.00120 

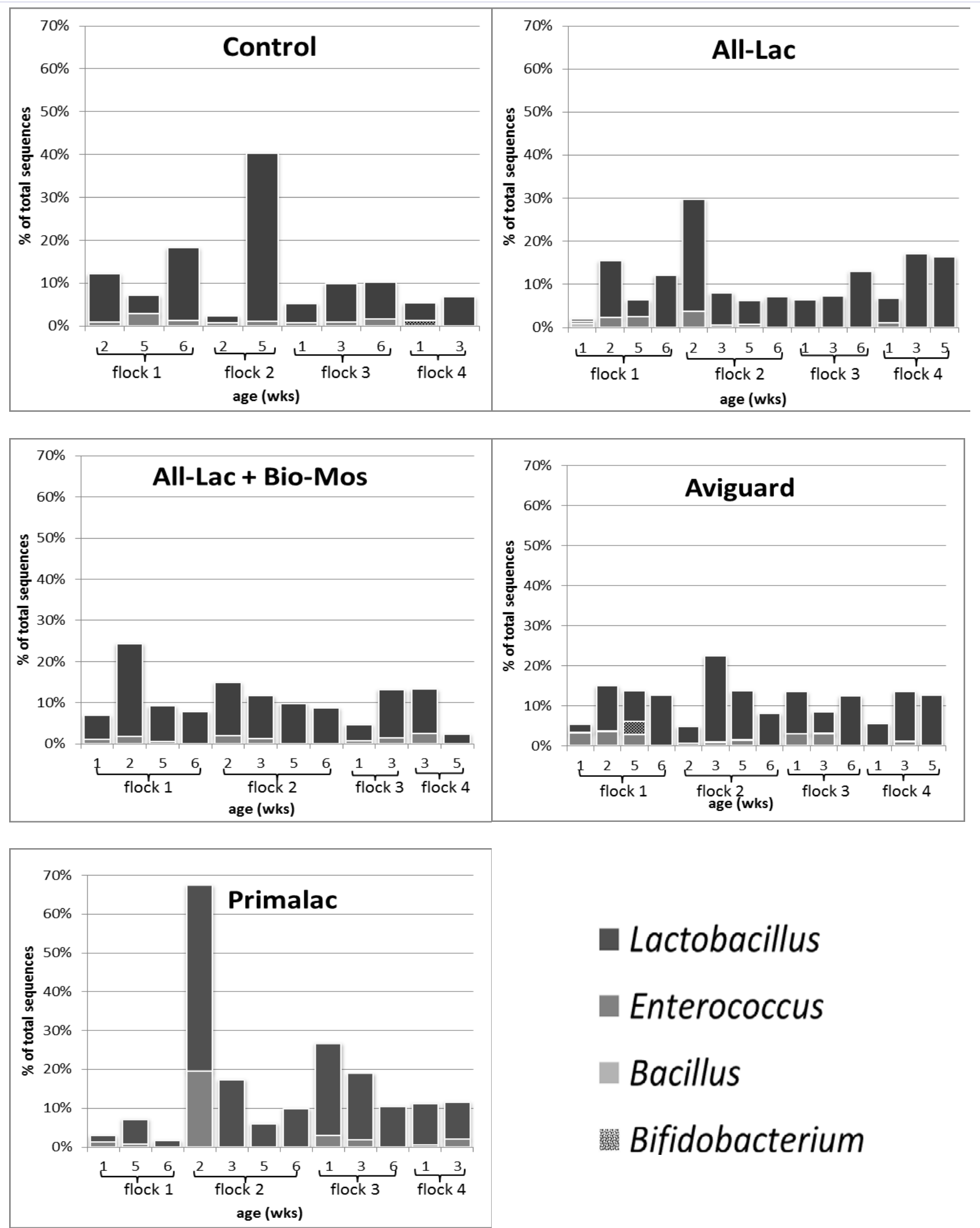

\section{Lactobacillus}

Enterococcus

\section{Bacillus}

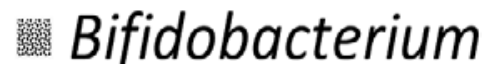

Figure 5: Effect of treatments on the abundance of commonly used probiotic bacteria within litter across multiple flock cycles. 
Table 1: Effects of treatments and time on the percentage of the microbiota represented by rare microbiome in broiler litter. Litter collected from birds receiving Primalact showed a higher percentage of the rare microbiome in the first flock compared to birds fed All-Lac + BioMos.

\begin{tabular}{|c|c|}
\hline Age (weeks) & Percentage \\
\hline 1 & 0.04 \\
\hline 2 & 0.04 \\
\hline 3 & 0.05 \\
\hline 5 & 0.03 \\
\hline 6 & 0.03 \\
\hline \multicolumn{2}{|c|}{ Flock } \\
\hline 1 & $0.04 \mathrm{AB}$ \\
\hline 2 & $0.03 \mathrm{AB}$ \\
\hline 3 & $0.03 \mathrm{~B}$ \\
\hline 4 & $0.05 \mathrm{~A}$ \\
\hline \multicolumn{2}{|c|}{ Treatment } \\
\hline Control & $0.04 \mathrm{~B}$ \\
\hline Primalac & $0.06 \mathrm{~A}$ \\
\hline All-Lac & $0.03 \mathrm{~B}$ \\
\hline All-Lac + Bio-Mos & $0.02 \mathrm{~B}$ \\
\hline Aviguard & $0.03 \mathrm{~B}$ \\
\hline \multicolumn{2}{|c|}{ Effects (Probability) } \\
\hline Age & NS \\
\hline Flock & 0.029 \\
\hline Treatment & 0.001 \\
\hline Treatment x Flock & 0.048 \\
\hline Treatment x Age & NS \\
\hline Age x Flock & NS \\
\hline
\end{tabular}

A, B. Significant differences are noted by different subscripts (A, B); NS = not significant.

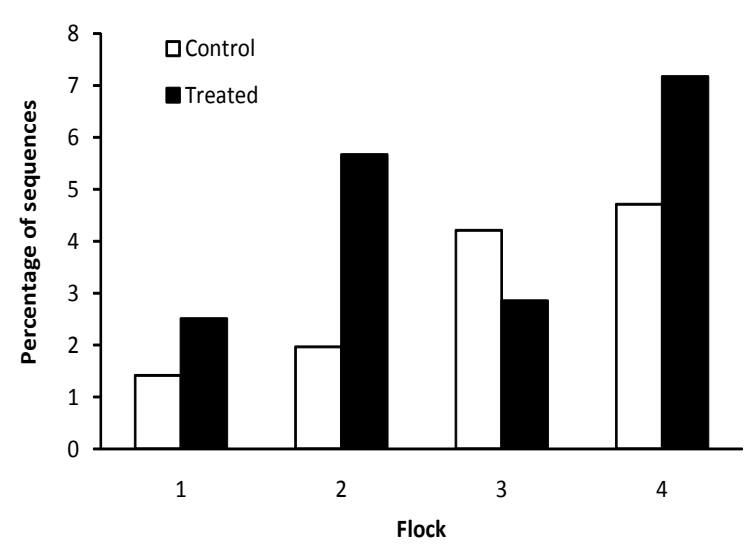

Figure 6: Abundance of putative pioneer colonizers in litter observed among flock cycles.

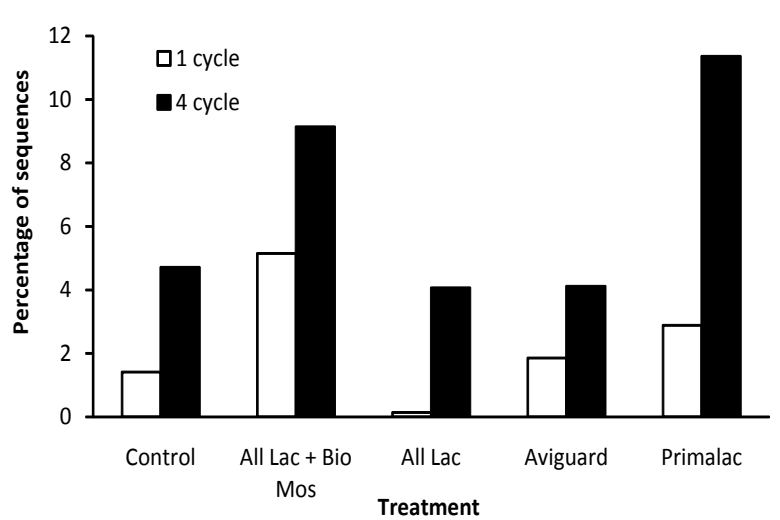

Figure 7: Abundance of pioneer colonizers in litter observed among treatments from first compared to last flock cycle.

producing bacterium SM4/1 were indeed increasing in abundance with flock cycles in the control group (Figure 6) but increased in abundance early on in litter from treated flocks (Figure 7). These microorganisms could be used as an indicator of the status of the litter and we propose that "good litter" is likely to have a higher proportion of rare biome that serves as a seed of pioneer colonizers for chicks.

The idea of using non pathogenic Clostridia species as pioneer colonizers in the microbiota is gaining momentum. For example, Clostridium butyricum has been shown effective as a poultry probiotic [48]. In addition, other butyrate producing bacteria such as Faecalibacterium prausnitzi, Roseburia faecis, and others may be associated with an healthy intestinal tract due the beneficial properties of this short chain fatty acid $[49,50]$. Some prebiotics also can improve the levels of $F$. prausnitzi in the intestine [51] and enrich the poultry litter. A litter with improved abundance of these organisms as pioneer colonizers could result in flocks with good intestinal health, better prepared to resist to pathogen colonization, and overall better animal performance.

\section{Effect of time and treatments on pathogen abundance}

Because deep litter systems are also known to contain pathogens, the litter sequence library was screened for the presence of bacterial pathogens of importance to poultry production. This analysis revealed that a number of potential pathogens were detected such as Arcanobacterium haemolyticum, Staphylococcus aureus, Clostridium perfringens and Erysipelothrix rhusiopathiae however their abundance was not affected by treatments or the cycle (Table 2). Abundance of Clostridium sordellii increased in litter overtime but treatments did not affect abundance. However, detection of Enterococcus cecorum presented a significant interaction between treatment and cycle (Table 3). Overall levels of E. cecorum decreased with cycling; however E. cecorum was more abundant in the control group in the second cycle than the treatments, indicating that antibiotic alternatives could affect the levels of this pathogen in reused litter after 1 cycle. 
Table 2: Abundance of sequences with homology to bacterial pathogens within the litter bacterial communities.

\begin{tabular}{|c|c|c|c|c|c|c|}
\hline \multicolumn{7}{|c|}{ Bacterial species (\% of total sequences) } \\
\hline Cycle number & $\begin{array}{l}\text { Arcanobacterium } \\
\text { haemolyticumt }\end{array}$ & $\begin{array}{l}\text { Staphylococcus } \\
\text { aureus }\end{array}$ & $\begin{array}{l}\text { Enterococcus } \\
\text { cecorum }\end{array}$ & $\begin{array}{l}\text { Clostridium } \\
\text { perfringens }\end{array}$ & $\begin{array}{l}\text { Clostridium } \\
\text { sordellii }\end{array}$ & $\begin{array}{l}\text { Erysipelothrix } \\
\text { rhusiopathiae }\end{array}$ \\
\hline 1 & 0.0140 & 0.0308 & $0.0196 \mathrm{AB}$ & 0.0041 & $0.4335 \mathrm{~A}$ & 0.0018 \\
\hline 2 & 0.0029 & 0.0114 & $0.0272 \mathrm{~A}$ & 0.0054 & $0.9589 \mathrm{AB}$ & 0.0019 \\
\hline 3 & 0.0000 & 0.0000 & $0.0053 \mathrm{~B}$ & 0.0159 & $1.3984 \mathrm{~B}$ & 0.0000 \\
\hline 4 & 0.0000 & 0.0000 & $0.0013 \mathrm{~B}$ & 0.0000 & $1.7037 \mathrm{~B}$ & 0.0000 \\
\hline \multicolumn{7}{|l|}{ Treatment } \\
\hline Control & 0.0000 & 0.0836 & $0.0505 \mathrm{~A}$ & 0.0116 & 0.8896 & 0.0000 \\
\hline All-Lac & 0.0061 & 0.0252 & $0.0000 \mathrm{~B}$ & 0.0062 & 1.1580 & 0.0000 \\
\hline $\begin{array}{l}\text { All-Lac + Bio- } \\
\text { Mos }\end{array}$ & 0.0040 & 0.1076 & $0.0114 \mathrm{AB}$ & 0.0015 & 0.8544 & 0.0000 \\
\hline Aviguard & 0.0011 & 0.0207 & $0.0181 \mathrm{AB}$ & 0.0068 & 0.9668 & 0.0024 \\
\hline Primalac & 0.0129 & 0.0000 & $0.0006 \mathrm{~B}$ & 0.0054 & 1.3415 & 0.0022 \\
\hline \multicolumn{7}{|l|}{ Probability } \\
\hline Cycle & NS & NS & 0.017 & NS & 0.018 & NS \\
\hline Treatment & NS & NS & 0.001 & NS & NS & NS \\
\hline $\begin{array}{l}\text { Cycle } \mathrm{x} \\
\text { treatment }\end{array}$ & NS & NS & 0.012 & NS & NS & NS \\
\hline CV\% & 358.1 & 220.2 & 251.5 & 348.7 & 89.1 & 590.9 \\
\hline
\end{tabular}

A,B. Significant differences are noted by different subscripts (A, B); NS = not significant.

Table 3: Effect of the interactions between sequential flock grow-out and treatment on Enterococcus cecorum abundance.

\begin{tabular}{|c|c|c|c|c|}
\hline Treatment & Cycle 1 & Cycle 2 & Cycle 3 & Cycle4 \\
\hline Control & $0.0653 \mathrm{~A}^{*}$ & $0.1803 \mathrm{aA}$ & $0.0000 \mathrm{~B}$ & $0.0000 \mathrm{~B}$ \\
\hline All-Lac & 0.0000 & $0.0000 \mathrm{~b}$ & 0.0000 & 0.0000 \\
\hline All-Lac + Bio-Mos & 0.0301 & $0.0000 \mathrm{~b}$ & 0.0084 & 0.0000 \\
\hline Aviguard & 0.0143 & $0.0302 \mathrm{~b}$ & 0.0194 & 0.0057 \\
\hline Primalac & 0.0000 & $0.0022 \mathrm{~b}$ & 0.0000 & 0.0000 \\
\hline
\end{tabular}

* Means followed by different capitalized letters (line comparisons) or lower letters in column comparisons indicate statistical differences by Tukey test $(\mathrm{P}<0.05)$.

\section{Conclusion}

Studies have demonstrated the adverse effects of used litter on performance in antibiotic-free poultry production [52]. This is particularly important because reduced use of antimicrobials correlates with shifting incidence of Enterococcus cecorum [53] and clostridial infections such as necrotic enteritis and gangrenous dermatitis [54]. Dumas used molecular ecology to compare litter microbiota from flocks with a history of gangrenous dermatitis and found that litter from wet houses had more gram-negative bacteria [47].

Cressman et al. reported that used litter produced more Clostridia within the ileum while fresh litter produced more environmental Lactobacillus [13]. In this study, we used molecular ecology to evaluate the composition of broiler litter over multiple flock cycles in order to reveal the effects of using antibiotic alternatives. Probiotics and prebiotics affected the overall composition of the litter microbiota as well as affecting the abundance of pathogens. We detected a reduction of Enterococcus in litter over subsequent flock cycles and in a previous report we described reduction of Salmonella as well [42]. Prebiotics have been shown to improve chick performance on new litter suggesting that they can affect prevalence of pathogens [44]. However in this study, we evaluate the abundance of putative pioneer colonizers in the litter in an effort to explain why multiple flock cycles are usually needed to realize full effects on gut health and performance. A number of reports have described the physiology of poultry litter in regards to nitrogen and carbon cycling including the major microbial groups involved in the processes $[37,46,55]$. But it is not known how these processes affect persistence or abundance of pathogens or of intestinal bacteria that may be important as pioneer colonizers for chicks. In this study we revealed that probiotics and prebiotics have the ability to alter litter microbiota. The poultry industries' understanding of the environmental effects of alternatives to antibiotics is in its infancy. Future research is needed to fully utilize these products in an effort to colonize chicks with a healthy 
microbiota and minimize the impact of pathogens encountered by today's commercial broiler chickens in the environment.

\section{Acknowledgments}

This work was supported by grant 2009-03561 to MDL from the National Institute of Food and Agriculture and the State of Georgia Veterinary Medical Experiment Station.

\section{References}

1. Schneitz C (2005) Competitive exclusion in poultry--30 years of research. Food Control 16(8): 657-667.

2. Kabir SM (2009) The Role of Probiotics in the Poultry Industry. Int. J. Mol. Sci 10(8):3531- 3546.

3. Gunal M, G Yayli, O Kaya, N Karahan, O Sulak (2009) The effects of antibiotic growth promoter, probiotic or organic acid supplementation on performance, intestinal microflora and tissue of broilers Int. J. Poult. Sci 5(2): 149-155.

4. Gustafson RH, Bowen RE (1997) Bowen Antibiotic use in animal agriculture J. Appl. Microbiol 83(5): 531-541.

5. Fairchild AS, JL Grimes, FT Jones, MJ Wineland, F Edens, et al. (2001) Effects of hen age, Bio-Mos, and Flavomycin on poult susceptibility to oral Escherichia coli challenge. Poult. Sci 80(5): 562-571.

6. Hooge DM (2004) Meta-analysis of broiler chicken pen trials evaluating dietary mannan oligosaccharide. Int. J. Poult. Sci 3(3): 163174.

7. Nurmi E, M Rantala (1973) New aspects of Salmonella infection in broiler production. Nature 241(5386): 210-211.

8. Waldroup PW, EO Oviedo-Rondon, CA Fritts (2003) Comparison of Bio-Mos and antibiotic feeding programs in broiler diets containing copper sulfate. Int. J. Poult .Sci 2: 3.

9. Fuller, R (1989) Probiotics in man and animals. J. Appl. Bacteriol 66(5): 365-378.

10. Metchnikoff E (1910) The prolongation of life. Heinemann.

11. Fuller R, R Fuller (1995) Probiotics: their development and use. In: Probiotics: Prospects of Use in Opportunistic Infections. PJ Heidt, V Rusch, D Van der Waaij, (Eds.), Institute for Microbiology and Biochemistry, Germany (8).

12. Patterson JA, KM Burkholder (2003) Application of prebiotics and probiotics in poultry production. Poult. Sci 82(4): 627-631.

13. Cressman MD, Z Yu, MC Nelson, SJ Moeller, MS Lilburn (2010) Interrelations between the microbiotas in the litter and in the intestines of commercial broiler chickens. Appl. Environ. Microbiol 76(19): 6572-6582.

14. Mead GC (2000) Prospects for 'competitive exclusion' treatment to control salmonellas and other foodborne pathogens in poultry. Vet J 159(2): 111-123.

15. Nava GM, LR Bielke, TR Callaway, MP Castaneda (2005) Probiotic alternatives to reduce gastrointestinal infections: the poultry experience. Anim. Health Res. Rev 6(1): 105-118.

16. Pedroso AA, JJ Maurer, Y Cheng, MDLee (2012) Remodeling the intestinal ecosystem toward better performance and intestinal health. The Journal of Applied Poultry Research 21:11.

17. Rantala M, E Nurmi (1973) Prevention of the growth of Salmonella infantis in chicks by the flora of the alimentary tract of chickens. Br.
Poult. Sci 14(6): 627-630.

18.Xu J, MK Bjursell, J Himrod, S Deng, LK Carmichael, et al. (2003) A genomic view of the human-Bacteroides thetaiotaomicron symbiosis. Science 299(5615): 2074-2076.

19. Xu J, JI Gordon (2003) Inaugural Article: Honor thy symbionts. Proc. Natl. Acad. Sci. USA 100(18): 10452-10459.

20. Asahara T, K Nomoto, K Shimizu, M Watanuki, R Tanaka (2001) Increased resistance of mice to Salmonella enterica serovar Typhimurium infection by symbiotic administration of Bifidobacteria and transgalactosylated oligosaccharides. J. Appl. Microbiol 91(6): 985-996.

21. Barthel M, S Hapfelmeier, L Quintanilla-Martinez, M Kremer, M Rohde, et al. (2003) Pretreatment of mice with streptomycin provides a Salmonella enterica serovar Typhimurium colitis model that allows analysis of both pathogen and host. Infect. Immun 71(5): 2839-2858.

22. Bohnhoff M, CP Miller, WR Martin (1964) Resistance of the Mouse's Intestinal Tract to Experimental Salmonella Infection. I. Factors Which Interfere with the Initiation of Infection by Oral Inoculation. J. Exp. Med 120(5): 805-816.

23. Hofacre CL, R Froyman, B George, MA Goodwin, J Brown (1998). Use of aviguard, virginiamycin, or bacitracin MD against Clostridium perfringens-associated necrotizing enteritis J. Appl. Poult Res 7(4).

24. Hofacre CL, AC Johnson, BJ Kelly, R Froymann (2002) Effect of a commercial competitive exclusion culture on reduction of colonization of an antibiotic-resistant pathogenic Escherichia coli in day-old broiler chickens. Avian Dis 46 (1): 198-202.

25. Hofacre CL, Primm ND, Vance K, MA Goodwin, J Brown (2000) Comparison of a lyophilized chicken-origin competitive exclusion culture, a lyophilized probiotic, and fresh turkey cecal material against Salmonella colonization. J. Appl. Poult. Res 9 (2): 195-203.

26. Isolauri E, Kirjavainen PV, Salminen S (2002) Salminen Probiotics: a role in the treatment of intestinal infection and inflammation? Br. Med J 50(3): 54-59.

27. Lee WJ (2008) Bacterial-modulated signaling pathways in gut homeostasis. Sci. Signal 1(21).

28. Lee YK, Menezes JS, Umesaki Y, Mazmanian SK (2011) Proinflammatory T-cell responses to gut microbiota promote experimental autoimmune encephalomyelitis. Proc Natl Acad Sci 108 (1): 4615-4622.

29. Menard S, Candalh C, Bambou J, Terpend K, Cerf-Bensussan N, et al. (2004) Lactic acid bacteria secrete metabolites retaining antiinflammatory properties after intestinal transport. Gut 53(6): 821828.

30. Neish AS (2010) Molecular Analysis of Microbiota-Host Cross-Talk in the Intestine. Biosci. Microflora 29: 1-10.

31. Sokol H, Pigneur B, Watterlot L, Lakhdari O, Bermúdez-Humarán LG, et al. (2008) Faecalibacterium prausnitzii is an anti-inflammatory commensal bacterium identified by gut microbiota analysis of Crohn disease patients. Proc. Natl. Acad. Sci 105(43): 16731-16736.

32. Walter J, Martinez I, Rose DJ (2013) Holobiont nutrition: Considering the role of the gastrointestinal microbiota in the health benefits of whole grains. Gut microbes 4(4): 340-346.

33. Griggs JP, Jacob JP (2005) Alternatives to Antibiotics for Organic Poultry Production J. Appl. Poult. Res 14(4): 750-756.

34. Floch, Martin H (2010) Fecal bacteriotherapy, fecal transplant, and the microbiome. J. Clin. Gastroenterol 44(8): 529-530. 
35. Collett SR (2012) The "Seed, Feed and Weed" approach to managing intestinal health in drug-free production systems. The Poultry Informed Professional $2013: 2-6$.

36. Cook KL, Rothrock MJ Jr, Warren JG, Sistani KR, Moore PA Jr (2008) Effect of alum treatment on the concentration of total and ureolytic microorganisms in poultry litter. J. Environ. Qual 37(6): 2360-2367.

37. Enticknap JJ, Nonogaki H, Place AR, Hill RT (2006) Microbial diversity associated with odor modification for production of fertilizers from chicken litter. Appl. Environ. Microbiol 72(6): 4105-4114.

38. Kelley TR, Pancorbo O, Merka W, Barnhart H (1998) Antibiotic resistance of bacterial litter isolates. Poult. Sci 77(2): 243-247.

39. Kelley TR, OC Pancorbo, WC Merka, SA Thompson, ML Cabrera, et al. (1995) Bacterial pathogens and indicators in poultry litter during reutilization. J. Appl. Poult. Res 4(4): 366-373.

40. Lu J, S Sanchez, C Hofacre, JJ Maurer, BG Harmon, et al. (2003) Evaluation of broiler litter with reference to the microbial composition as assessed by using $16 \mathrm{~S}$ rRNA and functional gene markers. Appl. Environ. Microbiol 69: 901-908.

41. Pedroso AA, AL Hurley-Bacon, AS Zedek, TW Kwan, AO Jordan, et al (2013) Can Probiotics Improve the Environmental Microbiome and Resistome of Commercial Poultry Production? Int. J. Env. Res. Public Health 10(10): 4534-4559.

42. Acosta Martinez V, S Dowd, Y Sun, and V Allen (2008) Tag encoded pyrosequencing analysis of bacterial diversity in a single soil type as affected by management and land use. Soil Biol \& Biochem 40(11): 2762-2770.

43. Schloss PD, SL Westcott, T Ryabin, JR Hall, M Hartmann, et al. (2009) Introducing mothur: open-source, platform-independent, community-supported software for describing and comparing microbial communities. Appl. Environ. Microbiol 75(23): 7537-7541.

44.SAS. Procedures Guide: Statistical Procedures. Cary. 2008.

45. Yang Y, PA Iji, A Kocher, LL Mikkelsen, M Choct (2007) Effects of Mannanoligosaccharide on Growth Performance, the Development of
Gut Microflora, and Gut Function of Broiler Chickens Raised on New Litter J. Appl. Poult. Res 16: 280-288.

46. Lovanh N, KL Cook, MJ Rothrock, DM Miles, K Sistani (2011) Spatial shifts in microbial population structure within poultry litter associated with physicochemical properties. Poult Sci 86: 1840-1849.

47. Dumas MD, S W Polson, D Ritter, J Ravel J Gelb, Jr R Morgan, et al. (2011) Impacts of poultry house environment on poultry litter bacterial community composition. PLoS One 6: (9).

48. Yang CM, GT Cao, PR Ferket, TT Liu, L Zhou, et al. (2012). Effects of probiotic, Clostridium butyricum, on growth performance, immune function, and cecal microflora in broiler chickens. Poult. Sci 91(9): 2121-2129.

49. Duncan SH, RI Aminov, KP Scott, P Louis, TB Stanton, et al. (2006) Proposal of Roseburia faecis sp. nov., Roseburia hominis sp. nov. and Roseburia inulinivorans sp. nov., based on isolates from human faeces. Int. J. Syst. Evol. Microbiol 56(10): 2437-2441.

50. Oikonomou G, AG Teixeira, C Foditsch, ML Bicalho, VS Machado RC, Bicalho (2013) Fecal microbial diversity in pre-weaned dairy calves as described by pyrosequencing of metagenomic $16 \mathrm{~S}$ rDNA. Associations of Faecalibacterium species with health and growth. PLoS One 8(4).

51. Haenen D, J Zhang, C Souza da Silva, G Bosch, I M van der Meer, et al. (2013) A diet high in resistant starch modulates micro biota composition, SCFA concentrations, and gene expression in pig intestine J. Nutr 143(3): 274-283.

52. Vieira SL, ET Moran (1999) Effects of delayed placement and used litter on broiler yields. J. Appl. Poult. Res 8(1): 75-81.

53. Armour NK, SR Collett, SM Williams (2011) Enterococcus cecorumrelated arthritis and osteomyelitis in broilers and broiler breeders. The Poultry Informed Professional (117).

54.Bedford, M (2000) Removal of antibiotic growth promoters from poultry diets: implications and strategies to minimise subsequent problems. Worlds Poult. Sci J 56(4): 347-365.

55. Nodar R, M Acea, T Carballas (1990) Microbial populations of poultry pine-sawdust litter. Biol. Wastes 33(4): 295-306. 